livraisons

d'Histoire

de l'Architecture

\section{Livraisons de l'histoire de l'architecture}

26 | 2013

Les ministres et les arts

\title{
Les ministres de l'Intérieur et les arts sous le Directoire
}

The Ministries of the Interior and the Arts under the Directoire

Die Innenminister und die Künste zur Zeit des Direktoriums

Marie-Claude Chaudonneret

(2) OpenEdition

Journals

Édition électronique

URL : http://journals.openedition.org/lha/334

DOI : $10.4000 /$ lha.334

ISSN : 1960-5994

Éditeur

Association Livraisons d'histoire de l'architecture - LHA

Édition imprimée

Date de publication : 10 décembre 2013

Pagination : 63-73

ISSN : $1627-4970$

Référence électronique

Marie-Claude Chaudonneret, «Les ministres de l'Intérieur et les arts sous le Directoire », Livraisons de I'histoire de l'architecture [En ligne], 26 | 2013, mis en ligne le 10 décembre 2015, consulté le 23 avril 2019. URL : http://journals.openedition.org//ha/334 ; DOI : 10.4000/lha.334 


\section{LES MINISTRES DE L'INTÉRIEUR ET LES ARTS SOUS LE DIRECTOIRE}

Le Directoire est, d'une façon générale, un moment trop ignoré ou sous-évalué. En matière d'institutions artistiques, on a trop tendance à penser qu'une administration moderne aurait été mise en place sous le Consulat, occultant ainsi la période précédente. Nous envisageons donc ici l'action du ministère de l'intérieur de la période post-thermidorienne au coup d'État de brumaire. Il ne s'agit pas de dresser un bilan complet de l'action du ministère mais d'envisager les débuts d'une nouvelle administration, les principales décisions et mesures décisives en faveur des arts, ainsi que les acteurs les plus remarquables de cette politique officielle.

Le décret du 12 germinal an II ( ${ }^{\text {er }}$ avril 1794) avait supprimé les ministères et les avait remplacés par douze commissions exécutives ; celles-ci étaient dirigées par plusieurs commissaires nommés par la Convention sur proposition du comité du Salut public. Ce mode de gouvernement avait engendré une inflation du personnel et une confusion des compétences entre commissions et comités. La Constitution de l'an III (22 août 1795), qui fonde le Directoire, supprime le système de gouvernement par des commissions exécutives et comités, reconstitue les ministères, lesquels se mettent en place en vendémiaire an 4 (septembre-octobre 1795). L'idée que le peuple possède le pouvoir de constituer l'État est abandonnée et on retrouve les six départements (dont celui de l'Intérieưr) de 1791. Par arrêté du Directoire exécutif du 12 brumaire an IV (3 novembre 1795) Pierre Bénézech est nommé ministre de l'intérieur; avant d'occuper cette fonction il avait été le 24 messidor an III (12 juillet 1795) l'un des commissaires de la commission des Armes puis était passé à la commission du Mouvement des armées de terre où il fut chargé de tout ce qui concernait le service de l'artillerie et du génie. Trop modéré, accusé d'amitiés royalistes, Bénézech est remplacé, le 27 messidor an V (15 juillet 1797), par Nicolas François dit François de Neufchâteau, commissaire du Directoire près le département des Vosges, républicain connu pour ses fortes convictions antiroyalistes. Deux mois après, François, élu membre du Directoire exécutif, est remplacé par François Sébastien Letourneux, commissaire près l'administration centrale du département de Loire inférieure. Éliminé par tirage au sort, François est rappelé au ministère de l'intérieur le 29 prairial an VI (17 juin 1798), ministère qu'il quitte le 4 messidor an VII (22 juin 1799). Bénézech et François ont pour eux la durée, cette stabilité leur permettant de mettre en place des actions cohérentes, ce qui n'avait pu être le cas lors de la période précédente. 


\section{Les débuts d'une administration moderne}

Les ministres de l'intérieur du Directoire entreprirent de rationaliser l'administration, de simplifier les structures. Le ministère fut alors constitué de six divisions : la première ayant la responsabilité des administrations des départements, la seconde des secours publics, la troisième des travaux publics, la quatrième de l'agriculture et du commerce, la cinquième de l'instruction publique, la sixième avait la charge de la comptabilité. De la cinquième division, qui prit rapidement le titre officiel de direction générale de l'Instruction publique, relevait l'instruction au sens large : écoles et bibliothèques, spectacles et fêtes, musées et Beaux-Arts ainsi que les poids et mesures. Avec cette réorganisation, se manifestait la volonté de centraliser le pouvoir de décisions, notamment en ce qui concernait les compétences du Musée central. À la fin de 1795 , la commission temporaire des Arts avait été supprimée en même temps que le comité d'Instruction publique : la dernière séance avait eu lieu le 20 décembre, le registre des procès-verbaux officiellement clos le 26 décembre. Deux jours avant, Bénézech avait réduit le nombre des membres de cette commis$\operatorname{sion}^{1}$, la réduction d'un personnel pléthorique faisant partie des mesures prises par le ministère. La commission temporaire des Arts fut remplacée par un conseil de Conservation, avec un personnel beaucoup moins nombreux que celui de la commission, et fonctionnant sous la tutelle du ministère de l'intérieur.

Bénézech avait réduit le personnel de certains bureaux, Neufchâteau les encadre plus fermement. Il exige une plus grande efficacité des chefs de bureaux placés sous son autorité. Il diffuse aux divers bureaux un "ordre du travail ", leur demandant de lui faire, tous les matins à 6 heures, un rapport, d'apporter le plus grand soin à la rédaction des lettres et rapports, qui doivent être extrêmement précis, clair et succinct en évitant "les phrases diffuses" et "les détails inutiles". Le ministre demande aux employés de bureaux qu'ils aient "l'esprit de leurs devoirs, l'amour de la patrie, l'horreur du royalisme, le respect pour la liberté ${ }^{2}$ ". Il exige d'eux qu'ils soient de bons patriotes; des enquêtes sont ordonnées pour connaître l'authenticité de leurs sentiments patriotiques. François entend également imposer son autorité, avoir plus d'autonomie par rapport au Directoire exécutif : "On élude indirectement la responsabilité du ministre, en faisant proposer au Directoire exécutif des arrêtés à prendre sur des objets que le ministre peut et doit même régler seul. C'est un abus qui surcharge le Directoire de détails administratifs mais le ministre entend faire à ses risques et périls, tous ce que les lois existantes l'autorisent à faire, et ne porter au Directoire que les grandes pensées et les résultats importants, faits pour

1. Bénézech, ministre de l'intérieur aux membres de l'ancienne commission temporaire des arts, 3 nivôse an IV, Arch. nat., $\mathrm{F}^{17} 1039 \mathrm{~B}$.

2. François, "Ordre du travail des bureaux du ministère de l'Intérieur ", 2 thermidor an VI ( 20 juillet 1798), Recueil des lettres circulaires, instructions, programmes, discours et autres actes publics émanant $d u C^{e n}$ François de Neufchâteau, Paris, an VII, t. 1, p. 1-11. 
fixer les yeux des premiers Magistrats ${ }^{3}$ ". C'est donc une administration efficace et relativement autonome que François met en place.

La nouveauté du ministère de l'intérieur sous le Directoire est la volonté de donner une place primordiale à l'instruction : alors que bien des attributions du ministère de l'intérieur sont héritières de l'Ancien régime, il a fallu inventer totalement une administration de l'Instruction publique, administration dont le rôle est de former de bons citoyens et bons patriotes. Après la chute de Robespierre le souci d'instruire dans une perspective politique et de cohésion sociale est très fort. Dans ce dispositif une attention particulière est portée aux arts et aux sciences. Alors que le Directoire exécutif venait de s'installer, Bénézech, à peine nommé ministre, diffuse un communiqué " au peuple français " où il s'engage à " ranimer l'industrie et le commerce ", à " donner une nouvelle vie aux arts et aux sciences, remettre l'ordre social ». Avant même le rétablissement des ministères, entre en scène après la chute de Robespierre un personnage-clé, Pierre-Louis Ginguené. Après avoir été emprisonné sous la Terreur, il est nommé le 26 fructidor an II (12 septembre 1794) premier adjoint de Garat (le deuxième adjoint est Clément de Ris) à la commission exécutive de l'Instruction publique puis commissaire (en remplacement de Garat) de cette commission. Le ministère de l'intérieur reconstitué, et alors que le ministre n'est pas encore désigné, Ginguené est nommé directeur général de l'Instruction publique, place qu'il occupe jusqu'en mars 1798 , date à laquelle il est remplacé par Venceslas Jacquemont. Ginguené, écrivain, avait obtenu sous l'Ancien Régime un emploi de commis au contrôle général des Finances. Après 1789, il embrasse les idéaux révolutionnaires, écrit pour La Feuille villageoise, journal créé en 1790 pour répandre l'instruction civique dans les campagnes, journal auquel contribua également le futur ministre François de Neufchâteau. Le 10 floréal an II (29 avril 1794), en pleine Terreur, Ginguené fonde, avec Andrieux, Say, Duval, Lebreton et Toscan, La Décade philosophique, littéraire et politique et écrit dans cette revue décadaire des articles sur la musique et la littérature ; vers $1795-1796^{4}$, il entreprit une importante Histoire littéraire d'Italie (9 volumes) publiée en 1811.

Avec Ginguené, d'autres hommes de La Décade eurent des responsabilités au sein de la commission de l'Instruction publique. Deux fondateurs de la revue dirigent le Bureau des musées: Joachim Lebreton comme chef et Amaury Duval comme sous-chef; Jean-Baptiste Chaussard, qui écrivit pour la même revue, est placé à la tête du bureau des Fêtes nationales. En brumaire an IV, le ministère de l'intérieur rétabli, Amaury Duval remplace Lebreton comme chef du bureau des Musées. Originaire de Rennes comme Ginguené, Amaury Duval donna dans La Décade, sous le pseudonyme de Polyscope, une critique des Salons de 1795 et de 1796. Ginguené, appelé à donner son opinion sur Amaury Duval, dont il semble avoir été très proche, note : "esprit très cultivé, ami des arts, homme de lettres, et

3. Ibid., p. 2.

4. Paolo Grossi, Pierre Louis Ginguené, historien de la littérature italienne, Berne, Peter Lang, 2006, p. 125. 
très républicain ${ }^{5}$ ". C'est donc le réseau de $L a$ Décade, réseau en partie breton ${ }^{6}$, qui préside aux destinées de l'Instruction publique. Les fondateurs et rédacteurs de La Décade étaient des républicains modérés, proches des Idéologues pour qui l'instruction était indispensable si l'on voulait former de bons républicains susceptibles de servir la patrie. La revue faisait régulièrement part des progrès, des sciences, des arts et des techniques, en donnait des analyses scientifiques. Il apparait donc logique que les hommes de $L a$ Décade aient obtenu des postes-clés à la direction de l'Instruction publique ; ils contribuèrent fortement à mettre en place les institutions républicaines sous le Directoire. Les deux personnalités les plus marquantes furent Ginguené et Amaury Duval, lequel resta en place pendant tout le Directoire, le Consulat et l'Empire.

\section{L'encouragement à l'art vivant}

Une des grandes préoccupations après Thermidor fut le sort des artistes, privés de ressources. Il fallait reconsidérer la politique d'encouragement à l'art vivant. Dans cette perspective, le Salon pouvait contribuer à redynamiser la production artistique et susciter l'émulation. Le Salon de 1793, le premier de la République, avait été organisé par les artistes composant la Commune générale des arts; le livret avait été conçu comme un simple guide pour le visiteur, les œuvres étant cataloguées suivant l'accrochage. Le premier Salon du Directoire, celui de l'an IV (1795), est organisée par la commission exécutive de l'Instruction publique ; le livret n'est plus conçu comme un guide pour le visiteur mais comme un inventaire des artistes vivants et de leurs œuvres sur une année. Aussi, Renou qui rédige le livret sur ordre de la Commission exécutive retient, contre la volonté du conservatoire du Musée central, l'ordre alphabétique des artistes. Le Salon est, certes, destiné à un large public mais il est aussi moyen de valoriser la production contemporaine française comme le souligne le Discours préliminaire du livret. Ce discours est une forme de manifeste en faveur des « artistes de la France» et pour une autre conception de la liberté dans le monde des arts. En 1792, des artistes réunis en Commune des Arts avaient réclamé des concours comme moyen le plus démocratique pour les commandes d'œuvres d'art mais les concours de l'an II organisés difficilement et ayant donné des résultats décevants, l'auteur du discours préliminaire donne des arguments qui seront toujours mis en avant après l'échec de concours comme moyen de commande publique (concours de 1830, de 1848...) : l'artiste " de mérite " ne se présente pas, "il attend qu'on l'appelle ".

Marc Gerstein, souligne que Vivant Denon, qui était hostile aux concours, avait avancé les mêmes objections, et il note que l'auteur anonyme de ce discours

5. État général des employés des bureaux du ministère de lintérieur, $5^{e}$ division, an VI. État établi par Ginguené à la demande (datée du 2 vendémiaire an VI) du ministre Letourneux, Arch. nat., $\mathrm{F}^{1 \mathrm{~b}} \mathrm{I} / 5$.

6. Ginguené et Duval étaient de Rennes, Lebreton de Saint-Méen. Voir Marc Regaldo, "La Décade, revue bretonne ", Annales de Bretagne, 1976, vol. 83, p. 605-606. 
ne pouvait être qu'un " haut fonctionnaire " ${ }^{7}$. Si le livret était rédigé par l'administration du Muséum qui recevait les œuvres, la partie "officielle " était le fait de l'administration de l'Instruction publique, celle-ci ayant la responsabilité de l'exposition. L'auteur de ce texte publié en tête du livret du Salon de 1795 ne peut donc qu'être le commissaire de la commission d'Instruction publique, c'est-à-dire Ginguené. Pour ce dernier, le principe d'égalité à l'origine de l'idée de concours publique n'est qu'illusoire, il est contraire à la liberté de créer : " les seuls concours, qui font éclore les merveilles, sont ceux où les Artistes, livrés à leur propre génie, libres dans le choix du sujet qu'ils traitent [...] travaillent avec liberté d'esprit, espérant exposer leurs travaux au public éclairé qu'ils prennent pour juge ". Les seuls concours vraiment utiles sont les expositions publiques où "le gouvernement pourra distinguer ceux que des succès constants tirent de la foule ". Ginguené conclut que le gouvernement doit être le véritable protecteur des artistes. Amaury Duval, dans son compte rendu du Salon de l'an $\mathrm{IV}^{8}$, va dans le sens de ce discours. Après avoir noté que le Salon est un "usage utile et qu'il faut toujours conserver ", le critique affirme que les artistes doivent "être solennellement jugés par le PEUPLE ", ce qui suscitera l'émulation et fera naître les talents contrairement aux " concours académiques, dans lesquels on est jugé que par quelques hommes, où l'on a que des prix pécuniaires à espérer ". Il semble bien que Ginguené ait donné une impulsion décisive à l'encouragement à l'art vivant. Il connaissait le monde des artistes et cherchait à leur venir en aide. Alors qu'il était à la tête de la commission exécutive de l'Instruction publique, il avait fait attribuer à un jeune élève de David, François Gérard, un atelier au Louvre: "C'est une marque de l'estime et des espérances que tes ouvrages ont fait concevoir ${ }^{9}$." Quelques mois plus tard, alors qu'il est directeur général de l'Instruction publique, il obtient que le peintre échappe à la réquisition : "c'est dans l'exercice de votre art que vous devez servir la République ${ }^{10}$ "; Ginguené cite dans cette lettre le "monument national", l'esquisse du 10 août. Quelques semaines plus tôt, Gérard s'était fait remarquer au Salon de l'an IV par une œuvre novatrice, Bélisaire, tableau qui, selon Amaury Duval, "mérite la palme : il est même fort au-dessus des meilleurs du salon ${ }^{11}$ ". Il semble que Ginguené eut une sincère affection pour Gérard, l'amour de l'Italie les rapprochant comme le suggère la lettre de Ginguené accompagnant son Histoire littéraire de l'Italie qu'il lui offre en espérant que cet ouvrage lui rappellera des "souvenirs de [sa] cara patria $^{12}$ ". Bien que ce soit le seul exemple que nous ayons de la sollicitude du

7. Marc Gerstein, "Denon et la politique des concours", Les Vies de Dominique-Vivant Denon, Daniela Gallo dir., Paris, 2001, p. 353-354.

8. "Première lettre de Polyscope sur les ouvrages de peinture, sculpture, etc., exposés dans le grand salon du Muséum ", La Décade philosophique, politique et littéraire, 30 vendémiaire an 4, p. 139140 .

9. Lettre de Ginguené à Gérard, 12 germinal an III/1/ ${ }^{\mathrm{er}}$ avril 1795, Henri Gérard, Correspondance de François Gérard, peintre d'histoire, Paris, 1867, p. 212.

10. Lettre du 27 nivôse an IV/17 janvier 1796, ibid., p. 212-213.

11. "Cinquième lettre de Polyscope... ", La Décade, 10 frimaire an IV, p. 415.

12. Lettre du 31 décembre 1815, Correspondance, op. cit. (note 9), p. 214. De mère italienne, Gérard avait passé les douze premières années de sa vie à Rome. 
directeur de l'Instruction publique envers les artistes, il semble bien que Ginguené suivit de près la carrière des artistes susceptibles de faire honneur à l'École nationale.

Bénézech, devenu ministre de l'intérieur, laissa probablement un certain pouvoir de décisions au directeur général de l'Instruction publique. À la suite de Ginguené, le ministre conçoit le Salon comme le noyau de l'encouragement aux artistes et décide de le rendre annuel. Le préliminaire au livret du Salon de l'an V (1796) défend et justifie la décision de rendre annuelle l'exposition officielle, certains l'ayant contestée; on redonne dans ce livret l'adresse de Bénézech "aux artistes de l'école française " du 9 floréal an V (28 avril 1796) : " ce serait trop peu pour l'activité française, pour le progrès et l'encouragement des arts, de les [les ouvrages des artistes] borner, comme ils l'étaient avant la Révolution, à une seule exposition publique en deux ans ". En effet, poursuit le ministre, les artistes sont beaucoup plus nombreux et "l'émulation va renaitre " avec l'organisation de la direction de l'Instruction publique. Il exhorte les " artistes-citoyens " à offrir chaque année des ouvrages pour " imposer à l'univers l'obligation d'admirer l'École française devenue fertile ». Les artistes ont une mission patriotique, ils doivent, avec de nouveaux chefs d'œuvre, poursuivre l'œuvre des grands anciens et ainsi démontrer la grandeur et la singularité de l'école nationale.

C'est le même souci de donner une impulsion aux arts qui conduit Bénézech à réorganiser l'Institut, établi par la Constitution de l'an III. Avant la réorganisation de l'Institut, avant même la nomination du ministre de l'intérieur, Ginguené avait, dans un rapport au comité de l'Instruction publique, affirmé combien la suppression de l'Académie de France à Rome faisait du tort aux arts et avait proposé de la rétablir et de réintégrer Suvée comme directeur ${ }^{13}$. Dans un Rapport sur l'organisation de l'institut national Bénézech défend les académies : "les académies, que l'Institut national est destiné à remplacer ne furent point, comme on l'a publié, des Établissements du Despotisme ", elles sont destinées "à réunir tous les hommes qui ont cultivé les sciences, les lettres et les Beaux-Arts de la manière la plus distinguée. C'est le sommet de l'édifice de l'Instruction publique ${ }^{14}$ ". Il propose le Muséum comme lieu des séances de l'Institut : "Cet édifice m'a paru sous tous les rapports digne de devenir le temple des sciences, des lettres et des arts ". Bénézech cherche à justifier le rétablissement des académies, certains ayant peut-être eu peur d'un retour en arrière, d'autant plus que venait d'être créée l'administration des Bâtiments civils (avatar de l'ancienne direction générale des Bâtiments). C'est probablement pourquoi il décide que les réunions des membres de l'Institut se tiendront au sein du Musée, établissement non contesté, ouvert au public en pleine Terreur.

13. Commission exécutive de l'Instruction publique. Rapport de Ginguené au comité d'Instruction publique, Arch. nat., $\mathrm{F}^{17} 1066$, dossier 5 .

14. Le ministre de l'intérieur au Directoire exécutif, Rapport sur l'organisation de l'Institut national, 3 frimaire an 4 (24 novembre 1795), Arch. nat., $\mathrm{F}^{17} 1094$. 


\section{L'institution muséale}

Le Musée est l'autre institution phare dans le système de l'Instruction publique. Bénézech consacre l'avenir des musées de la République, en premier lieu le Musée des monuments français. Alexandre Lenoir avait ouvert le dépôt dont il était le gardien en août 1793 et avait eu rapidement l'idée de transformer ce dépôt en " musée historique "; c'est le 29 vendémiaire an IV (21 octobre 1795) que Ginguené, à la tête du comité d'Instruction publique, accepte le projet d'un Muséum particulier de Monuments français; le 19 germinal an IV (8 avril 1796) le ministre de l'intérieur, Bénézech, prend officiellement la décision de la création d'un Musée des Antiquités et monumens français; des fonds lui sont accordés. Lenoir, dans l'avant-propos de son catalogue de l'an VIII, dit sa gratitude aux fonctionnaires de l'administration de l'Instruction publique : " le citoyen Ginguené, et les chefs de cette division du ministère m'ont prodigieusement encouragé, et si j'ai obtenu les suffrages du public, c'est à ces amis des sciences et des arts auxquels j'en suis redevable ${ }^{15}$ \#. Si Bénézech donne l'impulsion décisive à ce musée national, il aménage également le Musée central des arts en entreprenant, dans la Grande Galerie, des travaux conduits sous la direction d'Hubert Robert. En prairial an IV, la Grande Galerie étant fermée, on transporte un maximum de tableaux dans le salon carré que l'on ouvre aux artistes et au public. Dans l'Avertissement du catalogue de cette exposition $^{16}$, le Conservatoire du muséum informe le lecteur sur la nature des travaux entrepris dans la Grande Galerie; il souligne que, pour se conformer au souhait des " amateurs ", on va faire l'essai de l'éclairage zéntithal et, si l'essai est concluant, il sera adopté pour l'ensemble de la Grande Galerie en cours de réfection, du moins quand "l'état des finances" le permettra. L'avertissement informe également que "suivant l'opinion la plus générale " les peintures seront classées par écoles : l'école française occupera les premières travées, puis, à la suite, seront présentées l'école flamande, et l'école italienne.

Au Musée central l'école nationale était représentée avec les peintures des autres écoles de peinture; parallèlement elle eut son propre musée, le Musée spécial de l'École française dans le château de Versailles, grande entreprise voulue par Bénézech. Dans une longue lettre datée du 22 pluviôse an V (20 janvier 1797), le ministre informe l'administration du Musée central des arts ${ }^{17}$ qu'elle a été réformée dans un but précis : "donner à nos richesses le relief convenable pour la splendeur de la république et de les disposer en même temps de la manière la plus utile pour les arts, et la plus glorieuse pour les artistes français ". Il souligne que le palais

15. Alexandre Lenoir, Description historique et chronologique des Monuments de sculpture réunis au Musée des monuments français, Paris, an VIII.

16. Notice des tableaux des trois écoles, choisis dans la Colléction du Muséum des Arts, rassemblés au Sallon d'exposition, pendant les travaux de la Gallerie, au mois de prairial an IV, Paris, an IV de la République.

17. Le ministre de l'intérieur à l'administration du Musée central des arts, 22 pluviôse an V, Arch. nat., $\mathrm{F}^{21} 569$. 
national de Versailles possède des "morceaux bien précieux " qui " ont leur place dans le Musée central" mais n'entend pas dépouiller Versailles qui offre "un superbe local pour la formation d'un grand musée ». Aussi, le ministre arrête que "le Musée spécial de l'École française serait établi dans les Grands Appartements de Versailles "; l'administration du Musée central et un jury d'artistes décideraient des œuvres à répartir dans les deux musées. On a souvent réduit ce musée de Versailles à un établissement destiné à abriter d'une part l'excédent des œuvres saisies, d'autre part les œuvres indignes de figurer comme des chefs-d'œuvre au Musée central. Les intentions à l'origine de ce musée sont nombreuses et plus complexes. Il fallait trouver une affectation au château de Versailles pour le sauver de la ruine ; en se réappropriant les Grands Appartements, en leur donnant une nouvelle destination on achevait de nationaliser l'ancien château royal. Par ailleurs, le ministre ne voulait pas "dépouiller une commune magnifique qui a tant perdu»; il fallait compenser la perte de quelques chefs-d'œuvre en créant une institution originale, un musée à la gloire des artistes français. Ce sont exactement ces arguments qui seront avancés plus tard, au début de la Restauration, pour la création du musée du Luxembourg (dédommager les pairs à qui on enlevait des œuvres majeures en créant un établissement à la gloire des artistes vivants français) ${ }^{18}$. A Versailles, il fut décidé de présenter, avec les peintures anciennes, des œuvres modernes : «le musée de l'École française serait incomplet si les artistes existants qui l'ont régénérée n'y avaient pas une place qu'ils ne peuvent obtenir de leur vivant au Musée central "; Bénézech ajoutait que les expositions publiques ne suffisent pas pour la gloire et l'émulation des artistes, argument qui fut également utilisé au moment de la création du Musée du Luxembourg. En introduisant des œuvres d'artistes vivants à la suite des œuvres anciennes, Bénézech reprenait d'une certaine façon les débats qui eurent lieu au moment de la formation du Musée central, débats sur la question de l'exposition, ou non, de peintures contemporaines avec les œuvres anciennes. Loin d'être un réceptacle d'œuvres secondaires (il présentait notamment vingt-deux Poussin dont les quatre Saisons), le musée de Versailles, en illustrant l'évolution de l'art pictural de la France depuis ses commencements jusqu'aux artistes vivants, était un monument à la gloire de l'école nationale.

L'Avertissement du catalogue de 1801 souligne que ce musée, consacré aux " seules productions du génie " des artistes français, devait honorer le pays et susciter "l'admiration des étrangers». Dans ce catalogue, pour les œuvres contemporaines, était donnée, sous le nom de l'artiste, la mention "peintre vivant ». Ces peintres vivants étaient au nombre de trente-six, depuis le plus âgé, et "réformateur » de l'école française, Vien, jusqu'à Prud'hon dont on pouvait voir une œuvre qui avait été exposée au Salon de 1799. Le musée national de Versailles, comme le musée d'histoire nationale de Lenoir, étaient les compléments nécessaires du musée universel du Louvre.

18. Marie-Claude Chaudonneret, "Le musée du Luxembourg, destiné aux artistes vivants ", L'État et les artistes. De la Restauration à la monarchie de Juillet, Paris, Flammarion, 1999, p. 30-36. 
Alors que le musée de Versailles était en cours de constitution, le conseil de conservation du Musée central, dans sa séance du 11 fructidor an VI (27 août 1798), suggère que l'immense quantité d'objets d'art relégués dans les dépôts puisse être répartie "avec équité entre les membres de la grande famille ": "Paris est la commune centrale des arts, il est juste qu'elle possède des chefs-d'œuvre en tout genre [...], les départements ont des droits incontestables à la jouissance des objets qui pour être moins précieux n'en méritent pas moins l'estime des connaisseurs ${ }^{19}$ ". Le fameux arrêté de Chaptal fondant les musées des départements est donc en germes trois ans auparavant. Des premiers envois ont eu lieu : avant les grands " envois " de Chaptal, le musée de Lyon reçut quelques œuvres en décembre 1798 , puis en mars $1799^{20}$.

\section{Les Fêtes : pédagogie et émulation}

Outre les musées et les écoles, l'administration de l'Instruction publique développe les fêtes, dans un souci de pédagogie et de cohésion sociale. Les fêtes sous le Directoire ne sont pas nouvelles, elles étaient régulières depuis la fête de la Fédération le 14 juillet 1790 . A partir de 1795, elles sont institutionnalisées. Leur organisation dépend du "bureau des Musées et Fêtes nationales", ce qui signifie bien que les fêtes ont le même enjeu que les musées. Avec François de Neufchâteau, c'est un véritable système qui se met en place, "le système morale de nos Fêtes ${ }^{21}$ "; sept fêtes nationales par an sont instaurées (fête de la fondation de la République, de la Jeunesse, des Époux, de la Reconnaissance, de l'Agriculture, de la Liberté, des Vieillards). Sous l'administration de Bénézech, on diffusait pour ces fêtes un simple programme ; avec François elles sont justifiées, elles deviennent objets d'instruction civique. À propos de la fête de la Jeunesse ${ }^{\circ} l e$ ministre, dans une circulaire aux administrations centrales et municipales, affirme qu' " une pensée philosophique a présidé à l'ordonnance du système des fêtes. Elles sont politiques ou morales. Les premières ont pour but de rappeler à l'universalité des citoyens, par des images imposantes, le sentiment de leur dignité, de leurs droits et de leurs devoirs; ou de solenniser des époques mémorables ${ }^{22}$ ".

Lors de la fête de la Liberté de thermidor an VI (27-28 juillet 1798) fut organisée l'« Entrée triomphale des objets de sciences et d'arts recueillis en Italie ", manifestation organisée en deux temps : réception des objets par le ministre de l'intérieur et les membres de l'Institut national puis, le deuxième jour, présentation de ces objets au Directoire exécutif ${ }^{23}$. Cette entrée triomphale précédait de un mois

19. Barbier et Naigeon, Rapport sur la nécessité et les moyens de répartir entre les départements le surplus des objets de sciences et d'art qui se trouvent à Paris, Conseil du Conservatoire, séance du 11 fructidor an VI, Arch. nat., $\mathrm{F}^{17}$ 1034, dossier 11.

20. Marie-Claude Chaudonneret, "Les origines du musée des beaux-arts de Lyon ", Bulletin des Musées et Monuments lyonnais, 1986, $\mathrm{n}^{\circ} 1, \mathrm{p} .79-95$.

21. Recueil..., op. cit. (note 2), t. 2, p. 232.

22. Ibid., t. 2, p. 106-107.

23. Ibid., t. 1, p. 12-18. 
l'ouverture du Salon et de deux mois l'Exposition des produits de l'industrie française. Cette dernière exposition fut organisée rapidement : le 9 fructidor an VI (26 août 1798) le ministre de l'intérieur envoie une circulaire aux départements où il annonce cette exposition qui devait avoir lieu les trois derniers jours complémentaires de l'an VI (19-21 septembre), sur le Champ de Mars, dans une sorte de temple, cet édicule qui avait reçu six semaines auparavant les fruits des conquêtes en Italie. Le ministre avait parfaitement conscience que, étant donné le peu de temps dont on disposait pour rassembler les objets, cette manifestation serait modeste. Le catalogue ${ }^{24}$ fut publié un an après et une note indique : "Les départements éloignés n'ayant pu être instruits des dispositions du Gouvernement en temps utile pour en profiter cette année, on a été privé des ressources nombreuses qu'aurait fournies l'industrie active de la plupart d'entre eux. " Si cette manifestation fut décidée rapidement et organisée en un mois, ce fut pour donner une suite, une sorte de pendant, à l'entrée triomphale des objets d'arts et de sciences qui avait apporté un faste supplémentaire à la fête de la Liberté. Dans sa circulaire aux départements, François de Neufchâteau, souligne que, lors de la Fête célébrant la fondation de la République, il convient de ne pas oublier "les arts utiles qui contribuent si puissamment à sa prospérité " : "La France républicaine est devenu l'asile des Beaux-Arts, et grâce au génie de nos Artistes et aux conquêtes de nos guerriers, c'est désormais dans nos Musées que l'Europe viendra en prendre des leçons. La Liberté appelle également les arts utiles, en allumant le flambeau d'une émulation inconnue sous le despotisme, et nous offre ainsi les moyens de surpasser nos rivaux et de vaincre nos ennemis ${ }^{25}$. " L'Exposition des produits de l'industrie avait un but économique, ranimer l'industrie et le commerce, mais aussi patriotique puisqu'il s'agissait de montrer les innovations techniques des manufactures françaises. C'était un défi porté à ceux qui affirmaient que la Révolution avait anéanti un savoir-faire et ruiné la France. Victorieuse sur les champs de bataille, victorieuse également sur le terrain artistique, la jeune République pouvait montrer ses produits industriels. Le "génie " français se manifestait à la fois dans ses produits manufacturés et dans sa production artistique (le Salon de 1798 avait été un grand succès).

À l'issue de l'Exposition des produits de l'industrie, des récompenses furent attribuées, pour susciter l'émulation, par un jury composé de neuf membres, des scientifiques et ouvriers (les chimistes Darcet et Chaptal, l'horloger Ferdinand Berthoud....), un peintre (Vien) et un sculpteur (Moitte). Plusieurs types de mérite furent récompensés : l'invention, le perfectionnement, l'utilité publique ; étaient exclues des récompenses les fabriques nationales de Sèvres et de Versailles, "attendu

24. Exposition publique des produits de l'Industrie. Catalogue des produits industriels qui ont été exposés au Champ de Mars pendant les trois derniers jours complémentaires de l'an VI; avec les noms, départements et demeures des artistes et manufacturiers qui ont concouru à l'exposition; suivi du procès-verbal du Jury nommé pour l'examen de ces produits, Paris, De l'imprimerie de la République, vendémiaire an VII.

25. Le ministre de l'intérieur aux administrations centrales de département, et aux commissaires du Directoire exécutif près de ces administrations, 9 fructidor an VI, Recueil..., op. cit. (note 2), t. 2, p. 102-105. 
que les encouragements qu'elles reçoivent du gouvernement leur donnent des moyens qu'il est difficile à des particuliers de réunir ". Le jury, dans le procèsverbal, notait que "dans les fabriques des voisins " rien n'était comparable avec ce que produisaient la manufacture d'étoffes Richard-Lenoir, l'imprimeur Didot, la manufacture de porcelaine Dihl et Guérhard, l'horloger Bréguet ou le fabricant de crayons Comté. En conclusion, le jury disait espérer que, avec cette nouvelle " institution à jamais mémorable ", commençait pour l'industrie française " une ère nouvelle ${ }^{26}$ ». Cette première exposition était modeste, mais l'impulsion était donnée; elle fut suivie par d'autres, deux sous le Consulat (1801 et 1802), une sous l'Empire (1806). À partir de 1819, les Expositions des produits de l'industrie sont organisées régulièrement et deviennent de plus en plus importantes; celle de 1798 avait présenté 110 ouvrages, celle de 1827 environ 17000 .

La France fut le seul pays à organiser ce genre de manifestation jusqu'aux années 1820. Le caractère pionnier de cette institution fut continuellement souligné, hommage fut rendu à François de Neufchâteau. Ainsi, après l'exposition de 1819, le chroniqueur des Annales de l'industrie ${ }^{27}$, souligne que " pour rendre honneur " à François, il ne suffit pas de dire "il a eu une belle idée " mais encore d'examiner la situation de l'époque. C'est dans le contexte de rivalités et de guerre entre la France et l'Angleterre que le ministre eut la hardiesse de faire appel «au patriotisme des manufacturiers, des artistes et des savans » et de poser " la première pierre du monument élevé à la gloire de l'industrie ". Sous la monarchie de Juillet, David d'Angers, dans un article sur les "expositions nationales ", met l'accent sur l'idée visionnaire de François de Neufchâteau (qu'il ne nomme pas) à l'origine de la première Exposition des produits de l'industrie. Pour l'artiste républicain " deux sortes d'expositions doivent intéresser particulièrement une nation : l'Exposition de l'industrie et celle des Beaux-Arts ${ }^{28}$ "; dans tne perspective pédagogique et patriotique, ces deux types d'exposition étaient " utiles ", complémentaires. David insiste ainsi sur un élément essentiel du système d'encouragement à la création, mis en place à l'orée du $\mathrm{XIX}^{\mathrm{e}}$ siècle et qui va perdurer pendant plusieurs décennies.

La période du Directoire apparaît bien comme un moment fondateur avec la mise en place d'un nouveau territoire institutionnel, l'instauration de relations nouvelles entre artistes, pouvoir politique et pouvoir intellectuel dont étaient issus les administrateurs ou ministres les plus remarquables, tels Pierre-Louis Ginguené et François de Neufchâteau.

26. Catalogue, op. cit. (note 24), p. 17-18 (Procès-verbal rédigé par les citoyens composant le jury [...] remis au ministre le $5^{e}$ jour complémentaire an VI).

27. "Des diverses expositions qui ont précédé celle de 1819, et des ministres qui les ont dirigées ", Annales de lindustrie nationale et étrangère, Paris, 1820, p. 53-57.

28. Pierre-Jean David d'Angers, "Expositions nationales", Dictionnaire politique-Encyclopédie du langage et de la science politique, Paris, 1842, p. 388-390. Ce document nous a été aimablement communiqué par Thierry Laugée. 